\title{
Diversity-Multiplexing Tradeoff and Outage Probability in MIMO Relay Channels
}

\author{
Georgy Levin and Sergey Loyka \\ School of Information Technology and Engineering, University of Ottawa \\ 161 Louis Pasteur, Ottawa, Ontario, Canada, K1N 6N5 \\ E-mail: \{glevin, sloyka\}@ site.uottawa.ca
}

\begin{abstract}
MIMO single-relay fading channels are studied, where the source and destination are equipped with multiple antennas and the relay has a single one. Compact closed-form expressions are obtained for the outage probability under i.i.d. and correlated Rayleigh-fading links. Insightful high-SNR approximations are derived, which show the impact of the number of antennas, correlation, relay noise, relaying protocol, etc. Diversity-multiplexing tradeoff (DMT) is obtained for a broad class of fading distributions, including, as special cases, Rayleigh, Rice, Nakagami, Weibull, which may be non-identical, spatially correlated and/or non-zero mean. The DMT is shown to depend not on a particular fading distribution, but rather on its polynomial behavior near zero. It turns out to be the same for the simple "amplify-and-forward" protocol and more complicated "decode-and-forward" one with capacity achieving codes, i.e. the full processing capability at the relay does not help to improve the DMT. However, we also emphasize significant difference between the SNR-asymptotic DMT and the finite-SNR outage performance: while the former is not improved by using an extra antenna on either side, the latter can be significantly improved and, in particular, an extra antenna can be traded-off for a full processing capability at the relay.
\end{abstract}

\section{INTRODUCTION}

Cooperative communication strategies have recently emerged as an efficient way to exploit multi-user diversity available in wireless networks to significantly improve their performance [1]. While the research was initially concentrated on the single-antenna systems [1][2], the emphasis has recently shifted towards multi-antenna systems [3][4]. Typical performance metrics in a fading channel include the outage capacity or the outage probability, mean (ergodic) capacity and error rates when specific codes/modulation formats are studied [1][5]. Due to the complexity of the analysis, the performance studies have been mainly limited to independent (but not necessarily identically distributed) Rayleigh (or Rician) fading channels [3]. The only exception is [5], where the outage probability/capacity has been studied for a generic fading distribution, but the analysis was limited to the lowSNR regime and the links are still required to be independent.

Since the MIMO systems present an additional level of difficulty in terms of performance evaluation, an elegant framework termed "diversity-multiplexing tradeoff" (DMT) has been proposed in [6], which allows one to quantify the system performance in terms of two principle gains, diversity and multiplexing, available in a slow-fading MIMO channel when $S N R \rightarrow \infty$. Many systems, for which the outage probability/capacity analysis is illusive, can be characterized and compared via their respective DMTs. This framework has been successfully applied to relay channels as well [3]. While the original DMT formulation of Zheng and Tse [6] is limited to i.i.d. Rayleigh fading channels, a generalization to a class of channels satisfying a number of conditions on the distribution function has been presented in [7]. While most of the DMTbased studies are limited to the $S N R \rightarrow \infty$ regime, the finiteSNR DMT of the relay channel with independent, Rayleighfading links and single-antenna terminals have been studied in [8].

In this paper, we consider the source and the destination of the relay channel equipped with multiple antennas and a relay node equipped with a single antenna (e.g. due to complexity constraints). We allow the fading to be non-identical, correlated Rayleigh or of generic distribution, and consider amplify-and-forward (AF) and decode-and-forward (DF) protocols.

The contributions of the paper are two-fold:

- The SNR-asymptotic DMT of the relay channels in the AF and DF modes is obtained for a broad class of fading distributions including, as special cases, Rayleigh, Ricean, Nakagami, and Weibull, which can be non-identical and/or spatially correlated (Theorems 1, 2 in Section III). The AF and DF systems have the same DMT, which depends on the minimum diversity order only.

- Outage probability for correlated and/or non-identical Rayleigh fading is obtained in a closed form (Theorems 3, 4 in Section IV) for any SNR. Its low-outage approximations (Corollaries 3.1, 4.1) reveal a number of insights, including the impact of correlation, number of antennas, protocols and relay noise. We emphasize that systems with the same DMT may have vastly different outage performance: while the DMT of the $1 \times 1,2 \times 1$ and $1 \times 2$ channels is the same, an additional antenna results in significantly lower outage probability or, equivalently, a significant SNR gain (about $10 \mathrm{~dB}$ at the outage probability $=10^{-3}$ ), not captured by the DMT framework (see Section V). An additional antenna can be traded off for the full processing capability at the relay. Moreover, an extra transmit rather than a receive antenna is preferable, since it helps to eliminate the negative impact of relay noise for the AF protocol.

\section{CHANNEL MODEL}

Consider a MIMO relay channel with a single relay node equipped with a single antenna (see Fig. 1); the source (transmitter) and the destination (receiver) have $m$ and $n$ antennas respectively. While we consider both the amplifyand-forward and decode-and-forward protocols, the former will be assumed for simplicity of exposition, unless indicated 
otherwise, with a fixed relay gain (this is motivated by the fact that it is simpler to implement). We assume no direct sourcedestination link. This is motivated by three reasons: (i) the direct link is usually much weaker than the relay one (e.g. no line-of-sight etc.), and thus can be neglected (this is the case when the relay link is needed most) [2]; (ii) the results obtained for the no direct link case can be used as a "building block" to analyze the direct link case; (iii) the direct link corresponds to the regular (no relay) systems and thus has been extensively studied and well-understood by now, the relay link, on the contrary, is not so well understood so we concentrate our attention on this case.

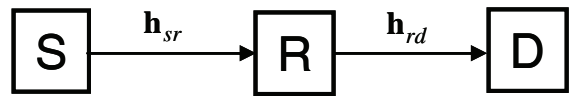

Fig. 1. Relay channel with a single relay node (R) (single-antenna) and multiple-antenna source (S) and destination (D).

The standard baseband system model of a frequency flat relay channel in the amplify-and-forward mode is,

$$
\mathbf{y}=\sqrt{G_{r d} G_{s r}} \mathbf{h}_{r d} \mathbf{h}_{s r}^{+} \mathbf{x}+\sqrt{G_{r d}} \mathbf{h}_{r d} \xi_{r}+\xi
$$

where $\mathbf{x}$ and $\mathbf{y}$ are the source (Tx) and destination ( $\mathrm{Rx}$ ) symbol vectors, $\mathbf{h}_{s r}$ and $\mathbf{h}_{r d}$ are the source-relay and relaydestination normalized channels (i.e. they include the multipath fading but not the average path loss), ${ }^{+}$denotes Hermitian conjugation, $G_{s r}$ and $G_{r d}$ are the source-relay and relay-destination average path loss factors, $\xi_{r} \sim \mathcal{C N}\left(0, \sigma_{r}^{2}\right)$ and $\xi \sim \mathcal{C N}\left(\mathbf{0}, \sigma_{0}^{2} \mathbf{I}\right)$ are relay and destination AWGN noise of variance $\sigma_{r}^{2}$ and $\sigma_{0}^{2}$ respectively, and independent of each other. A fixed relay gain $K_{r}$ is absorbed into $G_{r d}$ via the substitution $K_{r} G_{r d} \rightarrow G_{r d}$. Unless otherwise indicated, we do not assume any particular fading distribution of $\mathbf{h}_{s r}$ and $\mathbf{h}_{r d}$, but only assume that $\mathbf{h}_{s r}$ and $\mathbf{h}_{r d}$ are independent of each other, and require that PDFs $f_{s}(x)$ and $f_{d}(x)$ of $\left|\mathbf{h}_{s r}\right|^{2}$ and $\left|\mathbf{h}_{r d}\right|^{2}$ behave polynomially near zero, which hold for a broad class of fading distributions including, as special cases, Rayleigh, Ricean, Nakagami and Weibull fading, i.e. our results hold for a generic fading channel. Note that the first term in (1) represents the signal received at the destination; the second and the third terms represent the relay noise propagated to the destination and the destination noise. The sufficient statistics for $\mathbf{y}$ is,

$$
z=\frac{\mathbf{h}_{r d}^{+}}{\left|\mathbf{h}_{r d}\right|} \mathbf{y}=\sqrt{G_{r d} G_{s r}}\left|\mathbf{h}_{r d}\right| \mathbf{h}_{s r}^{+} \mathbf{x}+\sqrt{G_{r d}}\left|\mathbf{h}_{r d}\right| \xi_{r}+\frac{\mathbf{h}_{r d}^{+}}{\left|\mathbf{h}_{r d}\right|} \xi
$$

The instantaneous SNR at the destination can be expressed as

$$
\gamma^{\prime}=\frac{G_{r d} G_{s d}\left|\mathbf{h}_{r d}\right|^{2} \mathbf{h}_{s r}^{+} \mathbf{R}_{x} \mathbf{h}_{s r}}{\sigma_{0}^{2}+G_{r d}\left|\mathbf{h}_{r d}\right|^{2} \sigma_{r}^{2}} \leq \frac{\left|\mathbf{h}_{r d}\right|^{2}\left|\mathbf{h}_{s r}\right|^{2}}{1+\alpha\left|\mathbf{h}_{r d}\right|^{2}} \gamma_{0}=\gamma,
$$

where $\mathbf{R}_{x}=E\left\{\mathbf{x x}^{+}\right\}$is the covariance matrix of the transmitted signal, $\alpha=G_{r d} \sigma_{r}^{2} / \sigma_{0}^{2}$ is the ratio of the average relay noise propagated to the destination to the destination noise, and $\gamma_{0}=G_{r d} G_{s r} \sigma_{x}^{2} / \sigma_{0}^{2}$ is the average SNR at the destination, $\sigma_{x}^{2}=\operatorname{tr} \mathbf{R}_{x}=E\left\{\mathbf{x}^{+} \mathbf{x}\right\}$ is the total transmitted power (at the source). The inequality in (3) follows from $\mathbf{h}_{s r}^{+} \mathbf{R}_{x} \mathbf{h}_{s r} \leq\left|\mathbf{h}_{s r}\right|^{2} \sigma_{x}^{2}$, and the equality is achieved when
$\mathbf{R}_{x}=\sigma_{x}^{2} \mathbf{h}_{s r} \mathbf{h}_{s r}^{+} /\left|\mathbf{h}_{s r}\right|^{2}$, i.e. beamforming from the source to the relay, $\mathbf{x}=s \cdot \mathbf{h}_{s r} /\left|\mathbf{h}_{s r}\right|$, where $s$ is the scalar transmitted symbol of the total power $\sigma_{x}^{2}$. This requires channel state information (CSI) at the source. When no such information is available, a sensible transmission strategy is isotropic, i.e. $\mathbf{R}_{x}=\sigma_{x}^{2} \mathbf{I} / m$. In this case, the instantaneous SNR at the destination is $\gamma^{\prime}=\gamma / m$, i.e. the source CSI brings in an $m$ fold SNR gain, but does not change the statistics of the instantaneous SNR otherwise and, therefore, the outage probability or outage capacity differ by a constant SNR shift and the diversity-multiplexing tradeoff is the same in both cases. The instantaneous channel capacity (in nats $/ \mathrm{s} / \mathrm{Hz}$ ) can now be expressed as $C=\ln (1+\gamma)$ and the outage probability, i.e. the probability that the channel cannot support the target rate $R$, is $P_{\text {out }}(R)=\operatorname{Pr}\{C<R\}^{1}$.

\section{DIVERSITY-MULTIPLEXING TRADEOFF}

Following [6], we define the diversity gain as

$$
d=-\lim _{\gamma_{0} \rightarrow \infty} \ln P_{\text {out }} / \ln \gamma_{0}
$$

and the multiplexing gain from

$$
r=\lim _{\gamma_{0} \rightarrow \infty} R / \ln \gamma_{0},
$$

The SNR-asymptotic DMT of the relay channel in (1) for a broad class of fading distribution can be characterized as follows:

Theorem 1: Consider the relay channel in (1) such that the PDFs $f_{s}(x)$ and $f_{d}(x)$ of $\left|\mathbf{h}_{s r}\right|^{2}$ and $\left|\mathbf{h}_{r d}\right|^{2}$ behave polynomially near zero, i.e. $f_{s}(x) \sim x^{d_{s}-1}, f_{d}(x) \sim x^{d_{d}-1}$ as $x \rightarrow 0$, where $d_{s}, d_{d}$ are the diversity gains (orders) of the source-relay and relay-destination links at $r=0$, and $f(x) \sim g(x)$ means that there are constants $0<A \leq B<\infty$, such that $A g(x) \leq f(x) \leq B g(x)$ for sufficiently small $x$. The DMT of this channel in the amplify-and-forward mode is

$$
d(r)=\min \left(d_{s}(r), d_{d}(r)\right)=\min \left(d_{s}, d_{d}\right) \cdot(1-r), 0 \leq r \leq 1
$$

where $d_{s}(r)=d_{s} \cdot(1-r), d_{d}(r)=d_{d} \cdot(1-r)$ are the SNRasymptotic DMTs of the source-relay and relay-destination links ${ }^{2}$.

Proof: see Appendix.

Note that Theorem 1 holds for a broad class of fading distributions in the relay channel, which include, as special cases, Rayleigh, Ricean, Nakagami, and Weibull, and which may be non-identically distributed and spatially correlated. It is thus a generalization of [[9], Theorem 1] (for single relay case), where $\mathbf{h}_{s r}$ and $\mathbf{h}_{r d}$ are assumed to be i.i.d. complex Gaussian. Note also that the noise at the relay node does not affect the DMT, i.e. the channel in (1) with $\xi_{r}=0$ has the same DMT as in (6).

Theorem 1 shows that the DMT is affected by the number of degrees of freedom available in the channel and not by a

\footnotetext{
1 When capacity-achieving codes are used, BER $\approx \frac{1}{2} P_{\text {out }}$, and the word/frame error rate $\approx P_{\text {out }}$.

2 The DMT in (6) corresponds to an on-frequency relay; if orthogonal transmissions are used on the S-R and R-D links, the $r \rightarrow 2 r$ substitution should be used in (6).
} 
particular fading distribution, as long as the definition of diversity gain in (4) makes sense. Since the conditions of Theorem 1 relate to the behavior near zero of the distributions of $\left|\mathbf{h}_{s r}\right|^{2}$ and $\left|\mathbf{h}_{r d}\right|^{2}$, not the individual vector entries, it is straightforward to see that a full-rank correlation does not change the DMT. Following the discussion in Section II, the lack of source CSI is equivalent to an $m$-fold SNR loss and, therefore, has no effect on the SNR-asymptotic DMT. The transmit beamforming in combination with QAM modulation is an example of a space-time code that achieves the DMT of the single-relay channel with the source CSI. When no such CSI is available, isotropic transmission in combination with QAM will achieve the DMT.

Consider now the DMT of the decode-and-forward singlerelay channel, assuming capacity achieving codes and complete decoding/encoding at the relay.

Theorem 2: Under the conditions of Theorem 1, the DMT of the decode-and-forward single-relay channel is given by (6)

Proof (sketch): Since the instantaneous capacity in this case is $C=\min \left\{C_{s r}, C_{r d}\right\}$, where $C_{s r}$ and $C_{r d}$ are the capacities of the source-relay and relay-destination links, $P_{\text {out }}$ is dominated by $\max \left\{P_{s r}, P_{r d}\right\}$, where $P_{s r(r d)}=\operatorname{Pr}\left\{C_{s r(r d)}<R\right\}$ are the outage probabilities of the source-relay and relaydestination links, as $\gamma_{0} \rightarrow \infty$. Based on this, (6) follows after some manipulations.

Therefore, the single-relay channel subject to fading from a broad class of distributions has the same SNR-asymptotic DMT in the amplify-and-forward and decode-and-forward modes, i.e. the full processing capability at the relay (to achieve the capacity) does not help. Does it however mean that one should not employ such a processing? For many fading channels (e.g. i.i.d. or full-rank correlated), $d_{s}=m$, $d_{d}=n$. Thus, another conclusion from Theorem 1 is that there is no point in using unequal number of antennas (e.g. no point to use more destination than source antennas), since the DMT is affected by $\min (m, n)$ only. Do these conclusions still hold at finite SNR? To answer these questions, we need to consider the outage probability and not just the SNRasymptotic DMT, which is done in the next section.

\section{OUTAGE PROBABILITY}

Since the finite-SNR analysis is not feasible for a generic fading distribution, in this section we consider Rayleighfading links (i.e. source-relay and relay-destination), which may be non-identical and/or correlated.

Theorem 3: Let $\mathbf{h}_{r d}$ and $\mathbf{h}_{s r}$ be mutually independent circular symmetric correlated Gaussian random vectors. When the eigenvalues of the source-relay and relay-destination link correlation matrices $\mathbf{R}_{s r(r d)}=E\left(\mathbf{h}_{s r(r d)} \mathbf{h}_{s r(r d)}^{+}\right)$are non-zero and distinct, the outage probability of the single-relay channel in the amplify-and-forward mode is

$$
P_{\text {out }}(x)=1-\sum_{j=1}^{m} \sum_{i=1}^{n} A_{j} B_{i} e^{-\alpha x / \lambda_{j}} \sqrt{\frac{4 x}{\lambda_{j} \eta_{i}}} K_{1}\left(\sqrt{\frac{4 x}{\lambda_{j} \eta_{i}}}\right)
$$

where $K_{1}(x)$ is the first order modified Bessel function of the second kind [12], $A_{j}$ and $B_{i}$ are the coefficients of a partial fraction decomposition given by $A_{k}=\prod_{i \neq k} \lambda_{k} /\left(\lambda_{k}-\lambda_{i}\right)$ and likewise for $B, \lambda_{j}$ and $\eta_{i}$ are the eigenvalues of $\mathbf{R}_{s r(r d)}$, and $x=\left(e^{R}-1\right) / \gamma_{0}$.

Proof (sketch): Using the notations in the proof of Theorem 1 , the outage probability is

$$
P_{\text {out }}(x)=\operatorname{Pr}\left\{g_{s} g_{d}<x\right\}=\int_{0}^{\infty} f_{s}\left(g_{s}\right) F_{d}\left(x / g_{s}\right) d g_{s},
$$

where $F_{d}(x)$ is the CDF of $g_{d}$. Integrating (8) and using similar steps as in the proof of [[10], Theorem 1], (7) follows.

As expected, $P_{\text {out }}(x)$ increases with $\alpha$. While (7) holds when eigenvalues are different and non-zero, zero eigenvalues should be simply excluded, i.e. $m$ and $n$ should be treated as the ranks of corresponding correlation matrices.

To get some insight, let us consider the low-outage regime.

Corollary 3.1: $P_{\text {out }}(x)$ in Theorem 3 in the low outage regime $x \rightarrow 0$ is dominated by the $\min (m, n)$ term and is given by:

$$
\begin{aligned}
P_{\text {out }}(x) & =a_{1} x^{m}+o\left(x^{m}\right), & & m<n \\
& =a_{2} x^{n}+o\left(x^{n}\right), & & m>n \\
& =\left(a_{3}+b_{3} \ln \frac{1}{x}\right) x^{m}+o\left(x^{m}\right), & & m=n
\end{aligned}
$$

where $a_{1}, a_{2}, a_{3}$ and $b_{3}$ are constants independent of $x$

$$
\begin{aligned}
& a_{1}=\frac{\alpha^{m}}{m ! \operatorname{det} \mathbf{R}_{s r}}+\frac{(-1)^{m+1}}{\operatorname{det} \mathbf{R}_{s r}} \sum_{k=1}^{m} \sum_{i=1}^{n} \frac{B_{i} \ln \eta_{i}}{\eta_{i}^{k}} D_{k, m}(\alpha), \\
& a_{2}=\frac{(-1)^{n+1}}{n !(n-1) ! \operatorname{det} \mathbf{R}_{r d}} \sum_{j=1}^{m} \frac{A_{j} \ln \lambda_{j}}{\lambda_{j}^{n}}, \\
& a_{3}=\frac{\alpha^{m}}{m ! \operatorname{det} \mathbf{R}_{s r}}+\frac{(-1)^{m+1}}{m !(m-1) ! \operatorname{det} \mathbf{R}_{r d}} \sum_{j=1}^{m} \frac{A_{j}}{\lambda_{j}^{n}}\left(\ln \lambda_{j}+\Psi_{m}\right)+ \\
&+\frac{(-1)^{m+1}}{\operatorname{det} \mathbf{R}_{s r}} \sum_{k=1}^{m} \sum_{i=1}^{n} \frac{B_{i} \ln \left(\eta_{i}\right)}{\eta_{i}^{k}} D_{k, m}(\alpha), \\
& b_{3}=\frac{1}{m !(m-1) ! \operatorname{det} \mathbf{R}_{s r} \cdot \operatorname{det} \mathbf{R}_{r d}}, \\
& D_{k, l}(\alpha)=\frac{(-1)^{l-k} \alpha^{l-k}}{(l-k) !(k-1) ! k !},
\end{aligned}
$$

$\Psi_{k}=\psi(k)+\psi(k+1)$, and $\psi(k)$ is the Psi (digamma) function [12].

Proof (sketch): Using the standard series expansion of $K_{N}(x)$ [12], and making use of the following properties of partial fraction decomposition,

$$
\sum_{k=1}^{n} A_{k}=1, \sum_{k=1}^{n} \frac{A_{k}}{\lambda_{k}^{i}}=0, i=1, \ldots, n-1,
$$

(9) follows after some straightforward but lengthy manipulations.

In a typical wireless system, the average path loss is about $50 \ldots 100 \mathrm{~dB}$ or more (unless the transmitter and the receiver are very close to each other), so that when the relay gain and $\sigma_{r}^{2} / \sigma_{0}^{2}$ are not too large, $\alpha<<1$. Motivated by this, we note 
that the channel in (1) and the outage probability in (7) are the same as those of the single-keyhole model [10][[11], Theorem 3.1] when $\alpha \rightarrow 0$, and (9) simplifies to

$$
\begin{aligned}
P_{\text {out }}(x) & =\frac{x^{m}}{m !(m-1) !} \frac{\ln \frac{1}{x}+b_{m}}{\operatorname{det} \mathbf{R}_{r d} \operatorname{det} \mathbf{R}_{s r}}+o\left(x^{m}\right), \quad m=n \\
& =\frac{x^{m}}{m !(m-1) !} \sum_{i=1}^{n} \frac{B_{i} \ln \eta_{i}}{\left(\eta_{i}\right)^{m}} \frac{(-1)^{m-1}}{\operatorname{det} \mathbf{R}_{s r}}+o\left(x^{m}\right), \quad n>m
\end{aligned}
$$

where $b_{m}=1 / m+2 \psi(1)$, and the $m>n$ case is obtained from the $n>m$ one via $m \leftrightarrow n$. (11) clearly shows that the outage probability increases with correlation, since $\operatorname{det} \mathbf{R}$ is maximum for uncorrelated channel and decreases with correlation.

While Theorem 3 applies to a correlated channel with distinct eigenvalues and does not include the case of the i.i.d. channel, the latter is covered by the following result.

Theorem 4: Let $\mathbf{h}_{r d}$ and $\mathbf{h}_{s r}$ be i.i.d. circular-symmetric zero-mean Gaussian random vectors. The outage probability of the single-relay channel in the amplify-and-forward mode is

$$
P_{\text {out }}(x)=1-\frac{2 e^{-\alpha x}}{(n-1) !} \sum_{k=0}^{m-1} \sum_{i=0}^{k} \frac{\alpha^{i} x^{(k+i+n) / 2}}{i !(k-i) !} K_{k-i-n}(\sqrt{4 x})
$$

Proof (sketch): Following the same steps as in the proof of Theorem 3.

Note that (12) is easy to evaluate numerically, since it contains finite sums and well-known special functions. Let us now consider the low-outage regime.

Corollary 4.1: $P_{\text {out }}(x)$ in Theorem 4 is dominated by the $\min (m, n)$ term in the low-outage regime $x \rightarrow 0$ and is given by:

$$
\begin{aligned}
P_{\text {out }}(x) & =\frac{x^{m}}{(m-1) !} \sum_{k=0}^{m} \frac{\alpha^{k}(n-m+k-1) !}{(m-k) ! k !}+o\left(x^{m}\right), \quad m<n \\
& =\frac{x^{n}(m-n-1) !}{n !(m-1) !}+o\left(x^{n}\right), \quad m>n \\
& =\frac{x^{m}}{(m-1) !}\left(\sum_{k=1}^{m} \frac{\alpha^{k}(k-1) !}{(m-k) ! k !}+\frac{\ln \frac{1}{x}+b_{m}}{m !}\right)+o\left(x^{m}\right), m=n
\end{aligned}
$$

Proof (sketch): (13) follows after some straightforward but lengthy manipulations using the standard series expansion of $K_{N}(x)$ [12].

As in the correlated channel, the unusual term $\ln \frac{1}{x}$, which has a profound impact on the outage probability when $m=n$, is present. Unlike the $m \leq n$ case, $P_{\text {out }}(x)$ in (13) does not depend on $\alpha$ when $m>n$, i.e. extra Tx antenna(s) make the effect of relay noise negligible at the low-outage regime and thus are beneficial, even though they do not improve the DMT. When $\alpha=0, P_{\text {out }}(x)$ is symmetrical with respect to $m$ and $n$, which is explained by the symmetry of the channel model in (1) in this case.

\section{EXAMPLES}

To obtain some insight, let us consider $1 \times 1,2 \times 1$ and $1 \times 2$ i.i.d. Rayleigh-fading channels. In these cases, using $x=\left(e^{R}-1\right) / \gamma_{0}$ and $R=r \ln \gamma_{0}$ at high SNR, (13) simplifies to

$$
\begin{aligned}
& P_{\text {out }, 1 x 1} \approx \frac{\alpha+(1-r) \ln \gamma_{0}}{\gamma_{0}^{1-r}}, \\
& P_{\text {out }, 1 x 2} \approx \frac{1+\alpha}{\gamma_{0}^{1-r}}, \\
& P_{\text {out }, 2 x 1} \approx \frac{1}{\gamma_{0}^{1-r}},
\end{aligned}
$$

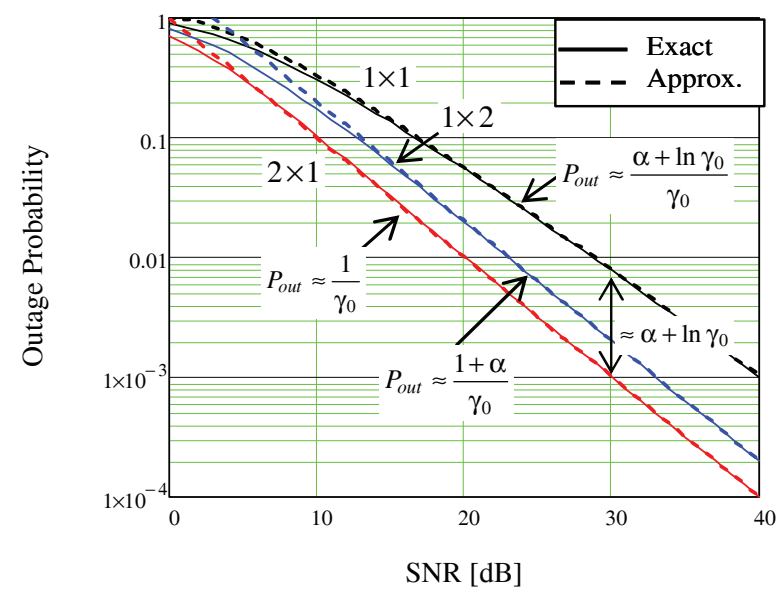

Fig. 2 Outage probability of $1 \times 1,2 \times 1$ and $1 \times 2$ i.i.d. Rayleigh-fading relay channels in the AF mode and their approximations in (14) for $\alpha=1$ and $r=0$ (this corresponds to fixed $R$ at high SNR). While the DMT is the same for all three channels, the gap between the outage probabilities increases with SNR: at $\mathrm{SNR}=40 \mathrm{~dB}$, the difference between $2 \times 1,1 \times 2$ and $1 \times 1$ channels is about $10 \mathrm{~dB}$ and even greater for larger $\alpha$.

Outage probabilities and their approximations are show in Fig. 2. It can be seen that the approximations are sufficiently accurate already for $\gamma_{0}>0 d B$. From (14),

$$
\frac{P_{\text {out }, 1 x 1}}{P_{\text {out }, 2 x 1}} \approx \alpha+(1-r) \ln \gamma_{0}, \frac{P_{\text {out }, 1 x 2}}{P_{\text {out }, 2 x 1}} \approx 1+\alpha
$$

so that $P_{\text {out }, 1 x 1} / P_{\text {out }, 2 x 1}$ and $P_{\text {out }, 1 x 1} / P_{\text {out }, 1 \times 2}$ grow unbounded as $\gamma_{0} \rightarrow \infty$ for $r<1$, i.e. there is a significant advantage in using an extra antenna at either Tx or Rx end at high SNR, even though the DMT is the same in all three cases (see Theorem 1). Moreover, (15) also shows that adding an extra Tx rather than $\mathrm{Rx}$ antenna is preferable for noisy relay.

When the relay node has full processing capability, i.e. the decode-and-forward protocol with capacity-achieving codes on both ends, the relay channel capacity is $C=\min \left\{C_{s r}, C_{r d}\right\}$, i.e. the weakest link dominates the outage performance, so that

$$
P_{\text {out }, 1 x 1} \approx \frac{2}{\gamma_{0}^{1-r}}
$$

assuming for simplicity the same average SNR on the Tx-relay 
and relay-Rx links. Comparing this to (14), we note that an extra Tx antenna in the AF mode is better then the full processing capability at the relay and can be used as a simple alternative of the latter, even though it does not improve the DMT (see Theorem 2). The same can be said about an extra $\mathrm{Rx}$ antenna if $\alpha \leq 1$. This indicates that the DMT framework is ill-suited for some channels and should be used with extreme caution when formulating design guidelines.

For the $1 \times 2$ and $2 \times 1$ channels in the DF mode,

$$
P_{\text {out }, 1 x 2}=P_{\text {out }, 2 x 1} \approx \frac{1}{\gamma_{0}^{1-r}}
$$

i.e. an extra antenna brings a modest $\mathrm{SNR}$ gain $(=3 \mathrm{~dB}$ at $r=0$ ), unlike the AF mode where this gain can be very significant (see Fig. 2). Comparing (17) to (14), we also note that the full processing capability at the relay does not bring in any advantage for the $2 \times 1$ channel; the same applies to the $1 \times 2$ channel when $\alpha<<1$.

\section{APPENDIX}

\section{Proof of Theorem 1:}

$$
\begin{array}{r}
\text { Let } g_{s}=\left|\mathbf{h}_{s r}\right|^{2}, g_{d}=\left|\mathbf{h}_{r d}\right|^{2} /\left(1+\alpha\left|\mathbf{h}_{r d}\right|^{2}\right) . \text { From (3) } \\
P_{\text {out }}(x)=\operatorname{Pr}\{C<R\}=\operatorname{Pr}\left\{g_{s} g_{d}<x\right\},
\end{array}
$$

where $x=\left(e^{R}-1\right) / \gamma_{0}$. For $R=r \ln \gamma_{0}, \quad 0 \leq r<1$, and $\gamma_{0} \rightarrow \infty, x \sim \gamma_{0}^{-(1-r)}$. Thus, $x \rightarrow 0$ as $\gamma_{0} \rightarrow \infty$. From (18),

$$
P_{\text {out }}(x)=\int_{0}^{\infty} f_{s}\left(g_{s}\right) F_{d}\left(x / g_{s}\right) d g_{s}=P_{1}+P_{2}+P_{3},
$$

where $F_{d}(x)$ is the CDF of $g_{d}$, and

$$
\begin{aligned}
& P_{1}(x)=\int_{0}^{x \ln \gamma_{0}} f_{s}\left(g_{s}\right) F_{d}\left(x / g_{s}\right) d g_{s} \\
& P_{2}(x)=\int_{x \ln \gamma_{0}}^{\delta} f_{s}\left(g_{s}\right) F_{d}\left(x / g_{s}\right) d g_{s}, \\
& P_{3}(x)=\int_{\delta}^{\infty} f_{s}\left(g_{s}\right) F_{d}\left(x / g_{s}\right) d g_{s}
\end{aligned}
$$

and $x \ln \gamma_{0}<\delta<<1, \quad \delta$ is a sufficiently small constant (independent of the SNR). In fact, $P_{1}(x)$ and $P_{3}(x)$ represent the outage events due to the outage of the source and destination terminals, respectively, and $P_{2}(x)$ represents the outage events due to simultaneous deep fades at both ends. The upper bound on $P_{1}(x)$ and $P_{3}(x)$ are

$$
\begin{aligned}
& P_{1}(x) \leq \int_{0}^{x \ln \gamma_{0}} f_{s}\left(g_{s}\right) d g_{s}=F_{s}\left(x \ln \gamma_{0}\right) \\
& P_{3}(x) \leq \int_{\delta}^{\infty} f_{s}\left(g_{s}\right) F_{d}(x / \delta) d g_{s}=\left(1-F_{s}(\delta)\right) F_{d}(x / \delta)
\end{aligned},
$$

where $F_{s}(x)$ is the $\mathrm{CDF}$ of $g_{s}$. Note that $F_{d}(x)=F_{r d}(x /(1-\alpha x))$ for $\alpha x \leq 1$ and 1 otherwise, where $F_{r d}(x)$ is the CDF of $\left|\mathbf{h}_{r d}\right|^{2}$. Therefore $F_{d}(x) \sim F_{r d}(x)$, Since $f_{s}(x) \sim x^{d_{s}-1} \quad$ and $\quad f_{d}(x) \sim x^{d_{d}-1}, \quad F_{s}(x) \sim x^{d_{s}} \quad$ and $F_{d}(x) \sim x^{d_{d}}$. Thus from (21),

$$
\begin{aligned}
& P_{1}(x) \leq F_{s}\left(x \ln \gamma_{0}\right) \sim\left(x \ln \gamma_{0}\right)^{d_{s}} \doteq x^{d_{s}} \\
& P_{3}(x) \leq\left(1-F_{s}(\delta)\right) F_{d}(x / \delta) \doteq x^{d_{d}}
\end{aligned}
$$

where $f_{1}\left(\gamma_{0}\right) \doteq f_{2}\left(\gamma_{0}\right)$ means exponential equality [1], [6]. Since $\delta$ is sufficiently small,

$$
\begin{aligned}
P_{2}(x) & =\int_{x \ln \gamma_{0}}^{\delta} f_{s}\left(g_{s}\right) F_{d}\left(x / g_{s}\right) d g_{s} \\
& \sim \int_{x \ln \gamma_{0}}^{\delta} g_{s}^{d_{s}-1}\left(x / g_{s}\right)^{d_{d}} d g_{s}=x^{d_{d}} \int_{x \ln \gamma_{0}}^{\delta} g_{s}^{d_{s}-d_{d}-1} d g_{s}
\end{aligned}
$$

If $d_{s} \neq d_{d}$,

$$
P_{2}(x) \sim \frac{x^{d_{d}}}{d_{d}-d_{s}}\left(\left(x \ln \gamma_{0}\right)^{d_{s}-d_{d}}-\delta^{d_{s}-d_{d}}\right) \doteq x^{\min \left(d_{s}, d_{d}\right)}
$$

If $d_{s}=d_{d}$,

$$
P_{2}(x) \sim x^{d_{s}}(\ln \delta-\ln (x \ln \gamma)) \doteq x^{d_{s}}
$$

Combining (22), (24), and (25), one obtains

$$
P_{\text {out }}(x) \doteq x^{\min \left(d_{s}, d_{d}\right)}=\gamma_{0}^{-\min \left(d_{s}, d_{d}\right) \cdot(1-r)}
$$

Applying (4), (6) follows Q.E.D.

\section{REFERENCES}

[1] N. Laneman, D. Tse, G. Wornell, "Cooperative Diversity in Wireless Networks: Efficient Protocols and Outage Behavior," IEEE Trans. Inform. Theory, vol. 50, no. 11, pp. 3062-3080, Dec. 2004.

[2] J. Boyer, D.D. Falconer, H. Yanikomeroglu, "Multihop Diversity in Wireless Relaying Channels," IEEE Trans. Communications, v. 52, no. 10, pp. 1820-1830, Oct. 2004.

[3] M. Yuksel, E. Erkip, "Multiple-Antenna Cooperative Wireless Systems: A Diversity-Multiplexing Tradeoff Perspective," IEEE Trans. Inform. Theory, vol. 53, no. 10, pp. 3371-3393, Oct. 2007.

[4] Y. Fan, J. Thompson, "MIMO Configurations for Relay Channels: Theory and Practice," IEEE Trans. Wireless Communications, vol. 6, no.5, pp.1774-1786, May 2007.

[5] G. Atia et al, "On Optimal Outage in Relay Channels With General Fading Distributions," IEEE Trans. Inform. Theory, vol. 53, no. 10, pp.3786-3797, Oct. 2007.

[6] L. Zheng, D. N. C. Tse, "Diversity and Multiplexing: A Fundamental Tradeoff in Multiple-Antenna Channels," IEEE Transactions on Information Theory, vol.49, no.5, pp. 1073-1096, May 2003.

[7] L. Zhao, W. Mo, Y. Ma, Z. Wang, "Diversity and Multiplexing Tradeoff in General Fading Channels," IEEE Transactions on Information Theory, vol.53, no.4, pp.1549-1557, April 2007.

[8] E. Stauffer et al, "Finite-SNR Diversity-Multiplexing Tradeoffs In Fading Relay Channels," IEEE JSAC, vol.25, no. 2, pp. 245-257, Feb. 2007.

[9] C. Rao, B. Hassibi, "Diversity-Multiplexing Gain Trade-off of a MIMO System with Relays," 2007 IEEE Information Theory Workshop on Information Theory for Wireless Networks, July 2007.

[10] G. Levin, S. Loyka, On the Outage Capacity Distribution of Correlated Keyhole MIMO Channels, IEEE Transactions on Information Theory, v. 54, no. 7, pp. 3232-3245, July 2008.

[11] G. Levin, Capacity Analysis of Asymptotically Large MIMO Channels, Ph.D. Thesis, University of Ottawa, Canada, 2008.

[12] I.S. Gradshteyn, I.M. Ryzhik, Tables of Integrals, Series and Products, Academic Press, San Diego, 2000. 